\title{
Article \\ Genome-Wide Association Study Identifies Eight Novel Loci for Susceptibility of Scrub Typhus and Highlights Immune-Related Signaling Pathways in Its Pathogenesis
}

\author{
Yong-Chan Kim ${ }^{1,2}$, Soriul Kim ${ }^{3}$, Hee-Kwon Kim ${ }^{4}$, Yi Lee ${ }^{5} \mathbb{D}$, Chol Shin ${ }^{3,6}$, Chang-Seop Lee ${ }^{7,8, *(\mathbb{D})}$ and \\ Byung-Hoon Jeong 1,2,*iD
}

Citation: Kim, Y.-C.; Kim, S.; Kim, H.-K.; Lee, Y.; Shin, C.; Lee, C.-S.; Jeong, B.-H. Genome-Wide Association Study Identifies Eight Novel Loci for Susceptibility of Scrub Typhus and Highlights Immune-Related Signaling Pathways in Its Pathogenesis. Cells 2021, 10, 570. https://doi.org/10.3390/ cells10030570

Academic Editor: Alexander E. Kalyuzhny

Received: 10 February 2021

Accepted: 1 March 2021

Published: 5 March 2021

Publisher's Note: MDPI stays neutral with regard to jurisdictional claims in published maps and institutional affiliations.

Copyright: (C) 2021 by the authors Licensee MDPI, Basel, Switzerland. This article is an open access article distributed under the terms and conditions of the Creative Commons Attribution (CC BY) license (https:/ / creativecommons.org/licenses/by/ $4.0 /)$.
1 Korea Zoonosis Research Institute, Jeonbuk National University, Iksan, Jeonbuk 54531, Korea; kych@jbnu.ac.kr

2 Department of Bioactive Material Sciences, Jeonbuk National University, Jeonju, Jeonbuk 54896, Korea

3 Institute for Human Genomic Study, College of Medicine, Korea University, Seoul 02841, Korea; soriul@korea.ac.kr (S.K.); chol-shin@korea.ac.kr (C.S.)

4 Molecular Imaging \& Therapeutic Medicine Research Center, Department of Nuclear Medicine, Biomedical Research Institute, Jeonbuk National University Medical School and Hospital, Jeonju, Jeonbuk 54907, Korea; hkkim717@jbnu.ac.kr

5 Department of Industrial Plant Science \& Technology, Chungbuk National University, Chungju, Chungbuk 28644, Korea; leeyi22@cbnu.ac.kr

6 Department of Internal Medicine, Division of Pulmonary Sleep and Critical Care Medicine, Korea University Ansan Hospital, Ansan 15355, Korea

7 Department of Internal Medicine, Research Institute of Clinical Medicine, Jeonbuk National University Medical School, Jeonju, Jeonbuk 54907, Korea

8 Biomedical Research Institute, Jeonbuk National University Hospital, Jeonju, Jeonbuk 54907, Korea

* Correspondence: lcsmd@jbnu.ac.kr (C.-S.L.); bhjeong@jbnu.ac.kr (B.-H.J.); Tel.: +82-63-250-2391 (C.-S.L.); +82-63-900-4040 (B.-H.J.); Fax: +82-63-254-1609 (C.-S.L.); +82-63-900-4012 (B.-H.J.)

Abstract: Scrub typhus is a fatal zoonotic disease caused by Orientia tsutsugamushi. This disease is accompanied by systemic vasculitis, lymphadenopathy, headache, myalgia, and eschar. In recent studies, a novel strain that is resistant to current medical treatment was identified in Thailand. Thus, the development of new specific drugs for scrub typhus is needed. However, the exact molecular mechanism governing the progression of scrub typhus has not been fully elucidated. To understand disease-related genetic factors and mechanisms associated with the progression of scrub typhus, we performed a genome-wide association study (GWAS) in scrub typhus-infected patients and found a scrub typhus-related signaling pathway by molecular interaction search tool (MIST) and PANTHER. We identified eight potent scrub typhus-related single nucleotide polymorphisms (SNPs) located on the PRMT6, PLGLB2, DTWD2, BATF, JDP2, ONECUT1, WDR72, KLK, MAP3K7, and TGFBR2 genes using a GWAS. We also identified 224 genes by analyzing protein-protein interactions among candidate genes of scrub typhus and identified 15 signaling pathways associated with over 10 genes by classifying these genes according to signaling pathways. The signaling pathway with the largest number of associated genes was the gonadotropin-releasing hormone receptor pathway, followed by the TGF-beta signaling pathway and the apoptosis signaling pathway. To the best of our knowledge, this report describes the first GWAS in scrub typhus.

Keywords: scrub typhus; Orientia tsutsugamushi; genome-wide association study; GWAS; MIST; PANTHER; signal pathway

\section{Introduction}

Scrub typhus is a zoonotic disease caused by Orientia tsutsugamushi, a Gram-negative family of Rickettsiaceae bacteria spread by chigger mites [1,2]. One billion people have been threatened by this disease globally, and more than one million new cases have been reported every year, mostly in the Asia-Pacific area [3,4]. Scrub typhus is an acute febrile 
disease accompanied by systemic vasculitis, lymphadenopathy, headache, myalgia, and eschar [5-7]. Since scrub typhus was first discovered in the 1930s in Japan, the extent of infection has been widening as global warming and globalization progress [2]. The fatality rates vary widely around a median mortality of $1.4 \%$ for treated patients and $6.0 \%$ for untreated patients [8]. The first treatment of scrub typhus is antibiotics, including doxycycline or azithromycin, but chloramphenicol is an alternative [9]. A novel strain that is resistant to doxycycline and chloramphenicol has been identified in Thailand; however, there is some controversy [10-13]. Thus, the development of new specific drugs for scrub typhus is needed. Moreover, the exact molecular mechanism of scrub typhus progression has not been fully elucidated to date.

Because scrub typhus is a Rickettsia disease, it is not surprising that several studies have noted Rickettsia-related innate immune response proteins [14]. In this context, an important study using the candidate gene approach has been performed to identify the pathogenesis of scrub typhus associated with molecular mechanisms [15]. Single nucleotide polymorphism (SNP) of the heat shock protein 70 (HSP 70) gene showed an association with clinical outcomes in intensive care unit patients with scrub typhus-induced sepsis [15]. In addition, the nonsynonymous SNP Asp299Gly of the toll-like receptor (TLR) 4 gene was significantly associated with susceptibility to scrub typhus [13]. Although association studies may help to characterize the relationship between disease and host genes [16-19], the investigation was only performed in immune-related genes, and scrub typhus-related specific molecular pathways have been elusive to date. In recent studies, genome-wide association study (GWAS), which is a high-throughput convenient genetic method to generate or reveal disease-related molecular mechanisms based on genome-wide compared data between case and control populations using highly integrated SNP chips, has been widely used [20-22].

Thus, in the present study, we performed a GWAS in scrub typhus-infected patients to develop novel candidate genes that contribute to the pathogenesis of scrub typhus. In addition, we analyzed linkage disequilibrium (LD) to evaluate whether scrub typhusrelated SNPs were in strong genetic linkage. Furthermore, we used a molecular interaction search tool (MIST, http:/ / fgrtools.hms.harvard.edu/MIST/) to find protein-protein interaction partners of candidate genes of scrub typhus [23]. Finally, we analyzed the signaling pathway and gene function of scrub typhus-related molecules by PANTHER (http:/ / www.pantherdb.org/tools/csnpScoreForm.jsp) [24].

\section{Materials and Methods}

\subsection{Ethical Statement}

All samples were obtained with informed consent under institutional review boardapproved protocols. All procedures performed in the present study were approved according to guidelines of the institutional review board of Jeonbuk National University Hospital and in accordance with the 1964 Helsinki declaration and its later amendments or comparable ethical standards (approval number: IRB No. CUH 2016-09-005). All the samples and related data were anonymized prior to investigation.

\subsection{Subjects}

Blood samples of 74 Korean patients with laboratory-confirmed scrub typhus were provided by Jeonbuk National University Hospital. The diagnosis of patients with scrub typhus was confirmed by an increase in the indirect immunofluorescence assay (IFA) IgM titer $\geq 1: 160$ against $O$. tsutsugamushi or a $\geq 4$-fold increase in the IFA titer $O$. tsutsugamushi, or when a positive reaction was observed in a nested polymerase chain reaction (PCR) targeting the $56 \mathrm{kDa}$ gene of $O$. tsutsugamushi. All patients showed mild symptoms and were diagnosed at an early stage. Genomic DNA was purified from $200 \mu \mathrm{L}$ of blood using a Blood Genomic DNA Isolation kit (Qiagen, Valencia, CA, USA) following the manufacturer's instructions. The GWAS data of 74 healthy Koreans were provided by Korea University. The exclusion criteria of healthy Koreans were asthma, chronic obstructive 
pulmonary disease, hepatitis, tuberculosis, head trauma, urinary tract infection, and cancer. The 74 healthy Koreans have no history of scrub typhus infection.

\subsection{GWAS}

Genomic DNA with 1.8-2.0 values of A260/A280 was used for genotyping. GWAS was performed by the Axiom ${ }^{\mathrm{TM}}$ Asia Precision Medicine Research Array (AMPRA) using a 500K SNP Chip (Thermo Fisher, Waltham, MA, USA). Genotyping was carried out using a Gaussian likelihood model. Quality control (QC) of genotyping data was performed, and SNPs with the following conditions were excluded: Hardy-Weinberg equilibrium (HWE) test, $p$ value $<1 \times 10^{-4}$; call rate $<95 \%$; minor allele frequency (MAF) $<1 \%$. Statistical analysis for identifying candidate SNPs was performed by Plink v1.90 (https:/ / www.coggenomics.org/plink2) and performed multivariate logistic regression models, including age and gender as covariates. Candidate SNPs with $p<0.0001$ were evaluated and excluded by Cluster QC. The plots were visualized by R v4.0.4 (https:/ / www.r-project.org/) and LocusZoom v0.12 (http:/ / locuszoom.org/). Candidate SNPs with $p$ value $<1 \times 10^{-3}$ were analyzed by linkage disequilibrium (LD) test, and LD block was visualized by Haploview v4.2 (Broad Institute, Cambridge, MA, USA). Statistical analyses were performed using SAS v9.4 (SAS Institute Inc., Cary, NC, USA). Statistical significance using $p$ value was calculated with a two-tailed Student's $t$ test and $\chi^{2}$ test.

\subsection{In Silico Analysis of Scrub Typhus-Related Candidate Genes and Signaling Pathways}

The biological interaction data were collected by the MIST database v5.0 (http:/ / fgrtools.hms.harvard.edu/MIST /) from model systems of several species, as well as humans. MIST can identify interacting partners based on protein-protein and genetic interaction data from the species of interest, as well as inferred interactions and visualize a corresponding network. PANTHER v16.0 (http:/ / www.pantherdb.org/tools / csnpScoreForm. jsp) is a comprehensive web-based platform for analyzing large-scale genome and gene function. PANTHER provides query gene functions and analyzes large-scale experimental data with a number of statistical tests.

\section{Results}

\subsection{Subjects}

A total of 148 individuals were included in the GWAS analysis. Detailed information on the study population is described in Table 1. A total of 74 scrub typhus-diagnosed patients were composed of 43 females and 31 males. A total of 74 healthy individuals were composed of 42 females and 32 males. The two groups showed a similar distribution of sex $(p=0.87)$. The mean age of diagnosis of scrub typhus patients was $64.4 \pm 7.6$ years, and the mean age of healthy individuals at sample collection was $64.4 \pm 7.6$ years. The mean ages between the two groups showed a very similar distribution $(p=0.93)$.

Table 1. Summary of information of subjects in this study.

\begin{tabular}{cccc}
\hline Characteristics & Case & Control \\
\hline Number & & 74 & 74 \\
Sex & Female & 43 & 42 \\
& Male & 31 & 32 \\
Mean age of diagnosis (years $\pm *$ STD) & $p$ value & $64.4 \pm 7.6$ & 0.87 \\
Mean age at sample collection (years $\pm *$ STD) & & & $64.4 \pm 7.6$ \\
& p value & & 1.0 \\
\hline *STD: Standard deviation & &
\end{tabular}

*STD: Standard deviation

\subsection{GWAS for Scrub Typhus}

GWAS was performed in QC and passed 477,093 SNPs using the Axiom ${ }^{\mathrm{TM}}$ AMPRA 500K SNP. A total of 1485 SNPs with $p<1 \times 10^{-4}$ values were refined by cluster QC, and 39 SNPs passed QC (Table 2). 
Table 2. Detailed information of single nucleotide polymorphisms (SNPs) analyzed in this study.

\begin{tabular}{ccc}
\hline$p$ Value $^{*}$ & Count & Cumulative \\
\hline$<1 \times 10^{-6}$ & 2 & 2 \\
$<1 \times 10^{-5}$ & 2 & 4 \\
$<1 \times 10^{-4}$ & 35 & 39 \\
$<1 \times 10^{-3}$ & 791 & 830 \\
$<0.01$ & 4219 & 5049 \\
$<0.05$ & 17,303 & 22,352 \\
$\mathrm{NS}^{* *}$ & 454,741 & 477,093 \\
\hline
\end{tabular}

* $p$ value indicates genetic difference of allele frequencies between case and control populations. ${ }^{* *}$ NS: Not significant.

Detailed information on candidate SNPs of scrub typhus with $p<1 \times 10^{-4}$ is described in Supplementary Table S1. Notably, four SNPs showed potent associations with $p<1 \times 10^{-5}$. The top SNP related to susceptibility of scrub typhus was the rs11184708 SNP, which is located upstream of the protein arginine methyltransferase 6 (PRMT6) gene with a $p=6.447 \times 10^{-13}$ value. The second SNP was the rs62140478 SNP, which is located upstream of the plasminogen-like B2 (PLGLB2) gene with a $p=7.404 \times 10^{-8}$ value. Rs2059950 of the DTW domain containing 2 (DTWD2) gene and rs74744256 of the one cut homeobox 1 (ONECUT1) and WD repeat domain 72 (WDR72) gene also showed a significant association of scrub typhus with $p<1 \times 10^{-5}$. A genomic overview of the association of susceptibility of scrub typhus was visualized by a Manhattan plot (Figure 1).

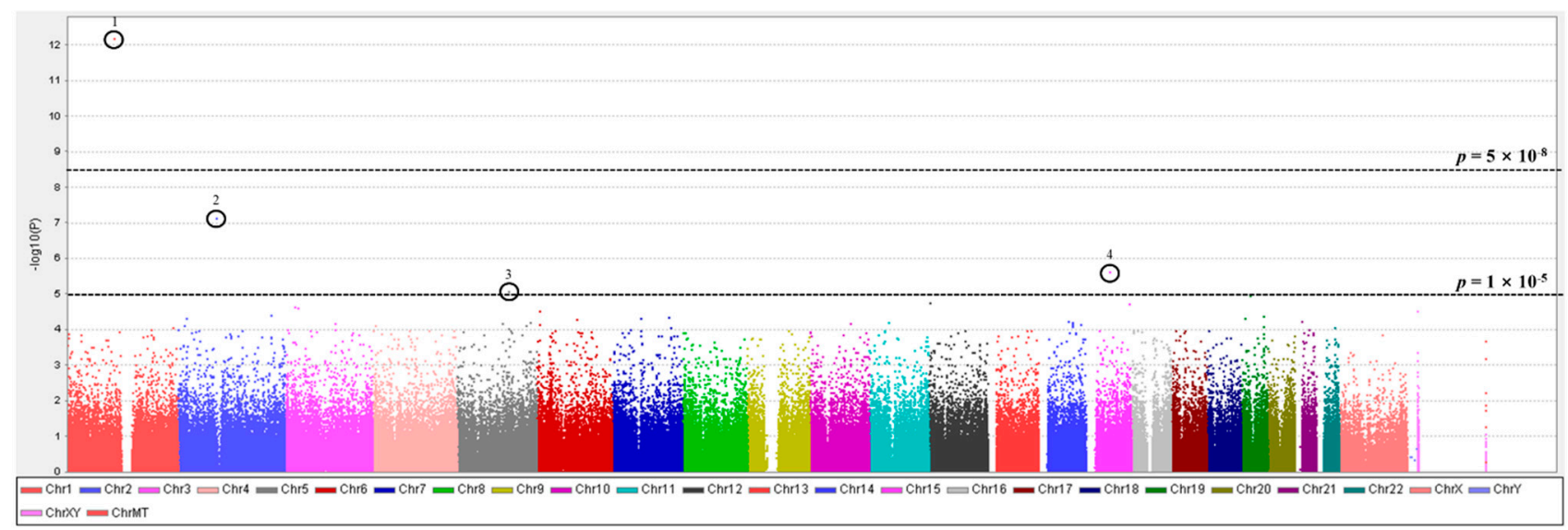

1: rs11184708; PRMT6

2: rs62140478; LOC101930107, MIR4435-1, PLGLB2

3: rs2059950; DTWD2, LOC102467225

4: rs74744256; ONECUTI, WDR72

Figure 1. Manhattan plot of the strength of association between scrub typhus-infected patients and healthy age- and sex-matched controls. $Y$ axis: $-\log 10(p)$ values; $X$ axis: chromosomal position. Dotted lines indicate two thresholds of potent association with $p$ value $=1 \times 10^{-5}$ and $p$ value $=5 \times 10^{-8}$.

Among candidate SNPs with $p<1 \times 10^{-4}, \mathrm{rs} 17103360$, rs7155418, and rs7155603 SNPs were located near the basic leucine zipper ATF-like transcription factor (BATF) and Jun dimerization protein 2 (JDP2) genes in chromosome 14. In addition, rs1654513 and rs2235091 SNPs were located near the kallikrein-related peptidase 4 (KLK4) gene in chromosome 19 (Supplementary Table S1). Regional plots were drawn by LocusZoom (Figure 2). 

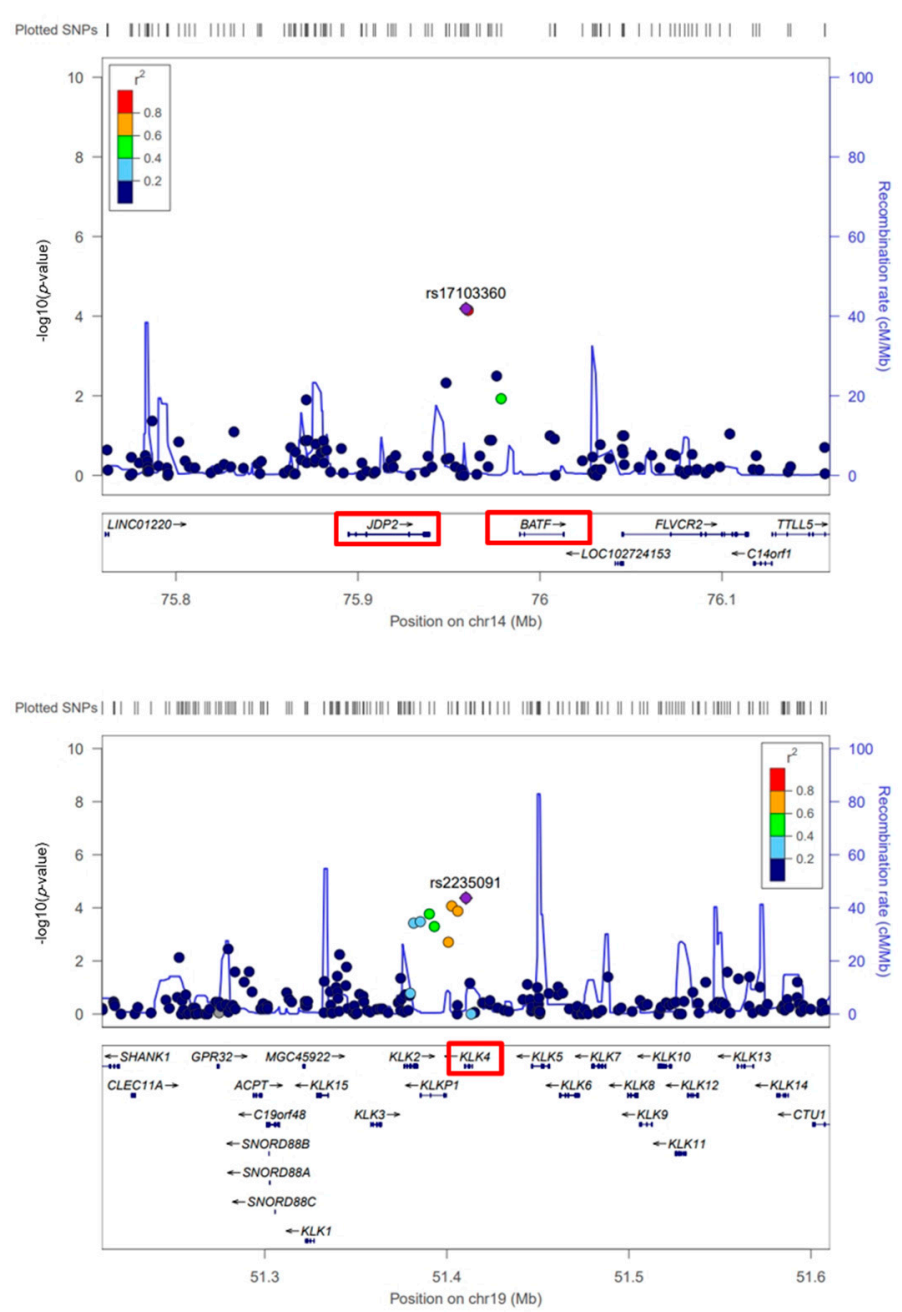

Figure 2. Illustration of association signals from strong linkage disequilibrium (LD) showed loci (top: JDP2 and BATF gene loci on chromosome 14; bottom: KLK4 gene locus on chromosome 19). Red boxes indicate the nearest genes with associated single nucleotide polymorphisms (SNPs). The diagram was visualized by LocusZoom (http://locuszoom.org/). AX-1282496: rs17103360; AX-12824299: rs7155418; AX-86334747: rs7155603; AX-40466275: rs198977; AX-11665883: rs8103659; AX-32775669: rs198956; AX-96079990: rs1354774; AX-11280320: rs1654513; AX-11663123: rs8103659; AX-40466329: rs2235091. The color indicates values of LD.

SNPs with $p<1 \times 10^{-3}$ that showed strong genetic linkage with regionally clustered SNPs with $p<1 \times 10^{-4}$ are listed in Supplementary Table S2. In addition, we analyzed strong LD blocks among these SNPs (Figure 3). Notably, three strong LD blocks were identified, including one LD block in chromosome 14 and two LD blocks in chromosome 19. The strong LD block in chromosome 14 was composed of three SNPs, including AX-1282496 (rs17103360), AX-12824299 (rs7155418), and AX-86334747 (rs7155603), and it was stretched approximately $1 \mathrm{~kb}$ (Supplementary Table S2, Figure 3A). Two strong LD blocks were found on chromosome 19. Of these two blocks, LD block 1 was composed of four SNPs, including AX-40466275 (rs198977), AX-11665883 (rs8103659), AX-32775669 (rs198956), and AX-96079990 (rs1354774), and was stretched approximately $11 \mathrm{~kb}$. LD block 2 was composed of three SNPs, including AX-11280320 (rs1654513), AX-11663123 (rs8103659) and AX-40466329 (rs2235091), and was stretched to $7 \mathrm{~kb}$ (Supplementary Table S2, Figure 3B). Next, we analyzed the signaling pathways of 39 candidate genes of scrub typhus with a $p$ value $<1 \times 10^{-4}$ using PANTHER (Supplementary Table S3). Notably, the mitogen-activated protein kinase kinase kinase 7 (MAP3K7) gene and TGF-beta receptor type-2 (TGFBR2) gene belong to the TGF-beta signaling pathway. 
(A)

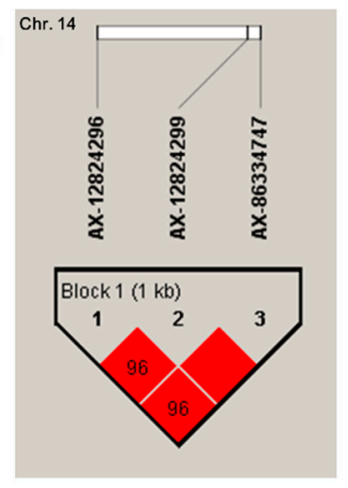

(B)

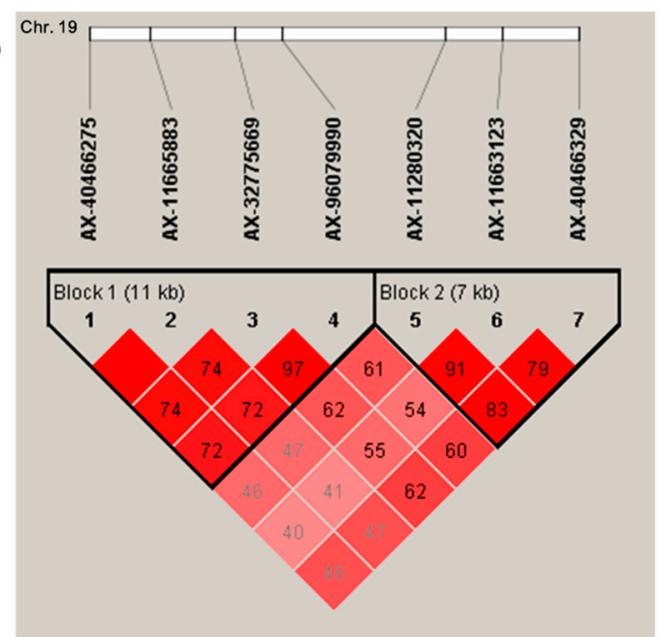

Figure 3. Linkage disequilibrium (LD) structure identified in chromosomes 14 and 19. (A) LD block located on the adjacent region of the JDP2 and BATF genes on chromosome 14. (B) LD blocks located on the adjacent region of the KLK4 gene on chromosome 19. The color gradient indicates values of LD.

\subsection{In Silico Analysis of Scrub Typhus-Related Candidate Genes and Signaling Pathways}

A total of eight candidate genes of scrub typhus, that is, PRMT6, DTWD2, BATF, JDP2, ONCUT1, KLK4, MAP3K7, and TGFBR2, with $p$ value $<1 \times 10^{-5}$, belonging to strong LD blocks or belonging to the same signal pathway, were analyzed by MIST to identify protein-protein interaction partners. A total of 224 genes predicted protein-protein interactions with candidate genes of scrub typhus were identified. The protein-protein interaction network of genes that interact with candidate genes of scrub typhus is visualized in Figure 4. We classified these genes that interact with candidate genes of scrub typhus according to the signaling pathway by PANTHER (Supplementary Table S4). A total of 62 signal pathways were identified. Among these signaling pathways, over 10 genes were associated with 15 signaling pathways, including angiogenesis, apoptosis signaling pathway, CCKR signaling map, EGF receptor signaling pathway, FGF signaling pathway, gonadotropin-releasing hormone receptor pathway, inflammation mediated by chemokine and cytokine signaling pathway, integrin signaling pathway, PDGF signaling pathway, Ras pathway, TGF-beta signaling pathway, Toll receptor signaling pathway, Wnt signaling pathway, and p53 pathway. Detailed information on signal pathway-related genes is shown in Supplementary Table S4. The signaling pathway with the largest number of associated genes was the gonadotropin-releasing hormone receptor pathway (31 genes) followed by the TGF-beta signaling pathway ( 25 genes) and the apoptosis signaling pathway ( 24 genes).

Finally, we investigated the immune-related function of genes that interact with candidate genes of scrub typhus based on protein-protein interactions. A total of five genes, including interleukin enhancer binding factor 2 (ILF2), 2'-5'-oligoadenylate synthetase 1 (OAS1), BATF, interferon-induced protein 35 (IFI35) and major histocompatibility complex, class II, DR alpha (HLA-DRA) genes, showed immune-related gene function. Detailed information on these genes is shown in Table 3. 


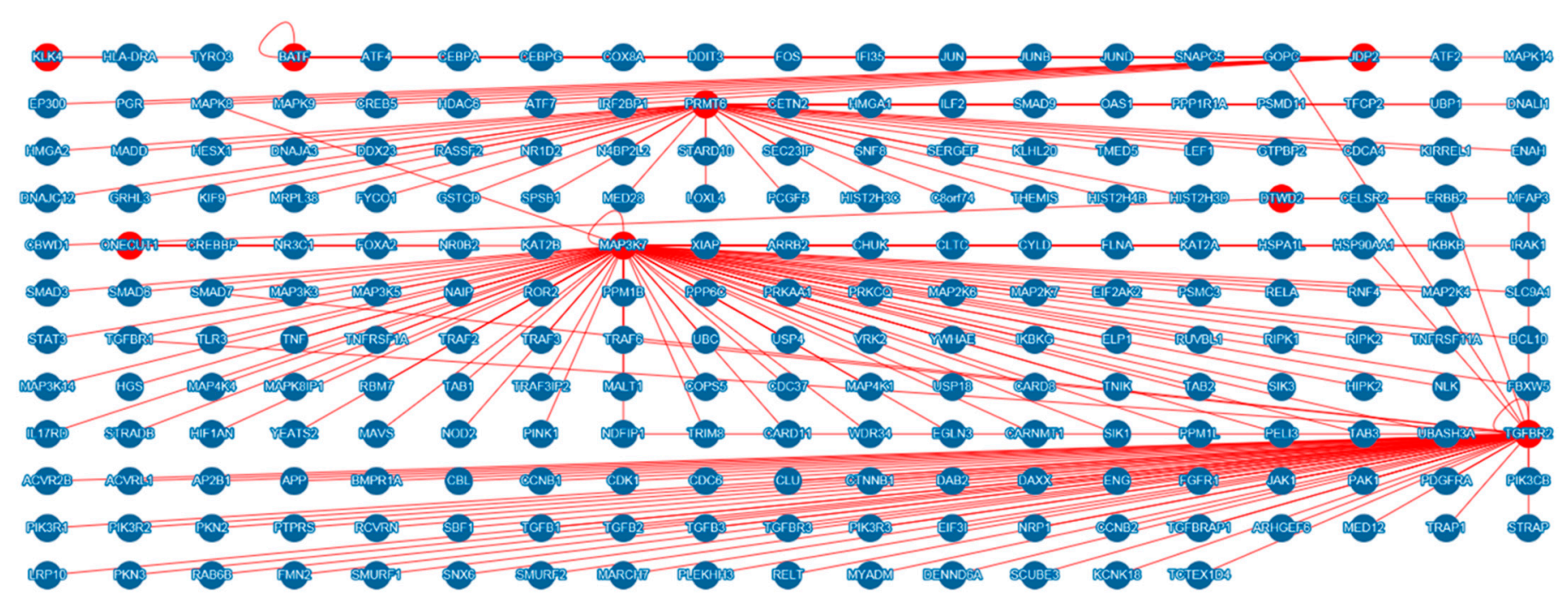

Figure 4. Protein-protein interaction genes with candidate genes of scrub typhus. Protein-protein interaction genes with candidate genes of scrub typhus were analyzed by MIST v5.0 (http:/ / fgrtools.hms.harvard.edu/MIST/) using grid layout. Red circles indicate candidate genes of scrub typhus. Red lines indicate protein-protein interactions.

Table 3. Immune-related genes that interact with candidate genes of scrub typhus based on protein-protein interaction.

\begin{tabular}{|c|c|c|c|}
\hline Gene & Gene Full Name & Function & $\begin{array}{l}\text { Related Candidate Gene of } \\
\text { Scrub Typhus }\end{array}$ \\
\hline ILF2 & Interleukin enhancer binding factor 2 & $\begin{array}{l}\text { Transcription factor required for T-cell expression } \\
\text { of the interleukin } 2 \text { gene }\end{array}$ & PRMT6 \\
\hline OAS1 & 2'-5'-oligoadenylate synthetase 1 & $\begin{array}{l}\text { Interferon-induced, dsRNA-activated antiviral } \\
\text { enzyme which plays a critical role in cellular } \\
\text { innate antiviral response }\end{array}$ & PRMT6 \\
\hline BATF & $\begin{array}{l}\text { Basic leucine zipper ATF-like } \\
\text { transcription factor }\end{array}$ & $\begin{array}{c}\text { AP-1 family transcription factor that controls the } \\
\text { differentiation of lineage-specific cells in the } \\
\text { immune system }\end{array}$ & - \\
\hline IFI35 & Interferon induced protein 35 & $\begin{array}{l}\text { IFI35 involved in interferon gamma signaling and } \\
\text { innate immune system }\end{array}$ & BATF, JDP2 \\
\hline$H L A-D R A$ & $\begin{array}{l}\text { Major histocompatibility complex, } \\
\text { class II, DR alpha }\end{array}$ & $\begin{array}{l}H L A-D R A \text { plays a central role in the immune } \\
\text { system by presenting peptides derived from } \\
\text { extracellular proteins }\end{array}$ & KLK4 \\
\hline
\end{tabular}

\section{Discussion}

In the present study, we carried out a genome-wide scale association study in scrub typhus patients for the first time. We identified a total of eight SNPs located on adjacent regions of the PRMT6, PLGLB2, DTWD2, BATF, JDP2, ONECUT1, WDR72, KLK, $M A P 3 K 7$, and TGFBR2 genes, which had a significant association with susceptibility to scrub typhus (Supplementary Table S1). Rs11184708, rs62140478, rs2059950, and rs74744256 showed significant associations with $p<1 \times 10^{-5}$. In addition, rs17103360 and rs2235091 showed a significant association with $p<1 \times 10^{-4}$, and adjacent SNPs showed strong LD (Supplementary Table S2) and composed LD blocks located on chromosomes 14 and 19. Furthermore, rs6809058 and rs16883596 belonged to the same signaling pathway. Because 10 candidate genes of scrub typhus were multifunctional genes, the role of the pathogenesis of scrub typhus was not determined. Thus, we analyzed the protein-protein interactions of candidate genes of scrub typhus. A total of 224 genes that interact with candidate genes of scrub typhus have been identified based on protein-protein interactions (Figure 4). To identify the scrub typhus-related signaling pathway, we classified 224 genes according to the signaling pathway. We postulated that multiple genes involved in the signaling pathway would be more affected by the scrub typhus-related signaling pathway. We identified 15 signal pathways involved in over 10 genes that interact with candidate genes of scrub 
typhus based on protein-protein interactions (Supplementary Table S4). The signaling pathway with the largest number of associated genes was the gonadotropin-releasing hormone receptor pathway. Since epidemiological studies reported that the susceptibility of women to scrub typhus was higher than that of men, further research investigating the association between susceptibility according to sex and the gonadotropin-releasing hormone receptor pathway is warranted [25]. TLR, which had been shown to be associated with susceptibility to scrub typhus in a previous study, also reaffirmed its association with the signaling pathway of scrub typhus [15]. Several immune-related signaling pathways, including the TGF-beta signaling pathway, Toll receptor signaling pathway, T cell activation, inflammation mediated by chemokine and cytokine signaling pathways, showed an association with scrub typhus. Further research on the CCKR signaling map, EGF receptor signaling pathway, FGF signaling pathway, PDGF signaling pathway, Wnt signaling pathway, and p53 pathway is needed in the future. Previous studies reported that the surface antigen of $O$. tsutsugamushi activates the nuclear factor-kB and p38 mitogen-activated protein kinase pathway and that intracellular invasion of $O$. tsutsugamushi is mediated by integrin signaling [26]. These signaling pathways were also identified in the present study (Supplementary Table S4).

Finally, we examined the function of genes that interact with candidate genes of scrub typhus based on protein-protein interactions. Five genes, including ILF2, OAS1, $B A T F$, IFI35, and HLA-DRA genes, showed apparent immune-related functions. The BATF gene located downstream of the JDP2 gene and adjacent GWAS SNP markers showed a significant association and composed a strong LD block. In addition, since the IFI35 gene is a downstream effector of these two genes, further investigation of the association of these genes with susceptibility to scrub typhus is highly desirable in the future. Furthermore, the ILF2 and OAS genes were downstream of the top SNP, and the rs11184708 SNP located upstream of the PRMT6 gene, which is related to susceptibility to scrub typhus. Further research investigating the relationship using overexpression and knockdown/out studies of these genes in cell culture and animal models of scrub typhus is warranted in the future.

However, since all scrub typhus patients showed mild symptoms and were diagnosed at an early stage, there may be bias in the data obtained. In addition, since the severity of infection ultimately could dictate the outcomes of the GWAS, including signal pathways and gene expression, further repetitive stratified GWAS analysis is highly desirable in patients showing various phenotypes, including the various degrees of symptoms and stages of infection.

\section{Conclusions}

In conclusion, in the present study, we performed GWAS in scrub typhus-infected patients. We identified eight potent scrub typhus-related SNPs located on the PRMT6, PLGLB2, DTWD2, BATF, JDP2, ONECUT1, WDR72, KLK, MAP3K7, and TGFBR2 genes. We analyzed protein-protein interaction genes with candidate genes of scrub typhus and identified 224 genes. We classified these genes according to signaling pathways and identified 15 signaling pathways associated with over 10 genes. The signaling pathway with the largest number of associated genes was the gonadotropin-releasing hormone receptor pathway. To the best of our knowledge, this report is the first systemic GWAS analysis to identify novel susceptible genetic biomarkers for scrub typhus infection.

Supplementary Materials: The following are available online at https:/ / www.mdpi.com/2073-4 409/10/3/570/s1, Table S1: Detailed information on scrub typhus-related candidate SNPs with a $p$ value $<1 \times 10^{-4}$; Table S2: Linkage disequilibrium (LD) of scrub typhus candidate SNPs with a $p$ value $<1 \times 10^{-3}$; Table S3: Signaling pathway of scrub typhus-related candidate SNPs with $p$ value $<1 \times 10^{-4}$; Table S4: Signaling pathways of genes that interact with candidate genes of scrub typhus based on protein-protein interactions. 
Author Contributions: Y.-C.K., C.-S.L., and B.-H.J. conceived and designed the experiment. Y.-C.K. and S.K. performed the experiments. Y.-C.K., S.K., H.-K.K., Y.L., C.S., C.-S.L., and B.-H.J. analyzed the data. Y.-C.K., C.-S.L., and B.-H.J. wrote the paper. All authors have read and agreed to the published version of the manuscript.

Funding: This study was supported by a Korea Centers for Disease Control and Prevention (KCDC) grant (No. 2013-E71005-00, 2014-E71003-00), a National Research Foundation of Korea (NRF) grant funded by the Korea government (MSIP) (NRF-2017R1A6A3A11034663), and a Korea University Grant. This research was supported by the Basic Science Program through the National Research Foundation (NRF) of Korea funded by the Ministry of Education, Science and Technology (2018R1D1A1B07048711). This research was supported by the Basic Science Research Program through the National Research Foundation (NRF) of Korea funded by the Ministry of Education (2017R1A6A1A03015876). This work was supported by NRF (National Research Foundation of Korea) Grant funded by the Korean Government (NRF-2019-Fostering Core Leaders of the Future Basic Science Program/Global Ph.D. Fellowship Program).

Institutional Review Board Statement: All samples were obtained with informed consent under institutional review board-approved protocols. All procedures performed in the present study were approved according to guidelines of the institutional review board of Jeonbuk National University Hospital and in accordance with the 1964 Helsinki declaration and its later amendments or comparable ethical standards (approval number: IRB No. CUH 2016-09-005). All the samples and related data were anonymized prior to investigation.

Informed Consent Statement: Informed consent was obtained from all subjects involved in the study.

Data Availability Statement: The data that support the findings of this study are available from the corresponding author upon reasonable request.

Acknowledgments: The biospecimens and data used in this study were provided by the Biobank of Jeonbuk National University Hospital, a member of the Korea Biobank Network, which is supported by the Ministry of Health, Welfare and Family Affairs. All samples derived from the Korea Biobank Network were obtained with informed consent under institutional review board-approved protocols (approval number: IRB No. CUH 2016-09-005).

Conflicts of Interest: The authors declare no conflict of interest, financial or otherwise.

\begin{tabular}{ll}
\multicolumn{2}{l}{ Abbreviations } \\
GWAS & Genome-wide association study \\
SNP & Single nucleotide polymorphism \\
HSP70 & Heat Shock Protein 70 \\
TLR4 & Toll-like receptor 4 \\
PRMT6 & Protein arginine methyltransferase 6 \\
ONECUT1 & One cut homeobox 1 \\
WDR72 & WD repeat domain 72 \\
BATF & Basic leucine zipper ATF-like transcription factor \\
JDP2 & Jun dimerization protein 2 \\
KLK4 & Kallikrein-related peptidase 4 \\
MAP3K7 & Mitogen-activated protein kinase kinase kinase 7 \\
TGFBR2 & TGF-beta receptor type-2 \\
ILF2 & Interleukin enhancer binding factor 2 \\
OAS1 & 2'-5'-oligoadenylate synthetase 1 \\
IFI35 & Interferon-induced protein 35 \\
HLA-DRA & Major histocompatibility complex, class II, DR alpha
\end{tabular}

\section{References}

1. Luce-Fedrow, A.; Lehman, M.L.; Kelly, D.J.; Mullins, K.; Maina, A.N.; Stewart, R.L.; Ge, H.; John, H.S.; Jiang, J.; Richards, A.L. A Review of Scrub Typhus (Orientia tsutsugamushi and Related Organisms): Then, Now, and Tomorrow. Trop. Med. Infect. Dis. 2018, 3, 8. [CrossRef] [PubMed]

2. Elliott, I.; Pearson, I.; Dahal, P.; Thomas, N.V.; Roberts, T.; Newton, P.N. Scrub typhus ecology: A systematic review of Orientia in vectors and hosts. Parasites Vectors 2019, 12, 513. [CrossRef] 
3. Kelly, D.J.; Fuerst, P.A.; Ching, W.M.; Richards, A.L. Scrub typhus: The geographic distribution of phenotypic and genotypic variants of Orientia tsutsugamushi. Clin. Infect. Dis. 2009, 48 (Suppl. 3), S203-S230. [CrossRef]

4. Xu, G.; Walker, D.H.; Jupiter, D.; Melby, P.C.; Arcari, C.M. A review of the global epidemiology of scrub typhus. PLoS Negl. Trop. Dis. 2017, 11, e0006062. [CrossRef]

5. Lee, C.S.; Hwang, J.H. IMAGES IN CLINICAL MEDICINE. Scrub Typhus. N. Engl. J. Med. 2015, 373, 2455. [CrossRef] [PubMed]

6. Koh, G.C.; Maude, R.J.; Paris, D.H.; Newton, P.N.; Blacksell, S.D. Diagnosis of scrub typhus. Am. J. Trop. Med. Hyg. 2010, 82, 368-370. [CrossRef]

7. Rajapakse, S.; Weeratunga, P.; Sivayoganathan, S.; Fernando, S.D. Clinical manifestations of scrub typhus. Trans. R Soc. Trop. Med. Hyg. 2017, 111, 43-54. [CrossRef]

8. Shrestha, P.; Roberts, T.; Homsana, A.; Myat, T.O.; Crump, J.A.; Lubell, Y.; Newton, P.N. Febrile illness in Asia: Gaps in epidemiology, diagnosis and management for informing health policy. Clin. Microbiol. Infect. 2018, 24, 815-826. [CrossRef] [PubMed]

9. El Sayed, I.; Liu, Q.; Wee, I.; Hine, P. Antibiotics for treating scrub typhus. Cochrane Database Syst. Rev. 2018, 9, CD002150. [CrossRef]

10. Watt, G.; Chouriyagune, C.; Ruangweerayud, R.; Watcharapichat, P.; Phulsuksombati, D.; Jongsakul, K.; Teja-Isavadharm, P.; Bhodhidatta, D.; Corcoran, K.D.; Dasch, G.A.; et al. Scrub typhus infections poorly responsive to antibiotics in northern Thailand. Lancet 1996, 348, 86-89. [CrossRef]

11. Kollars, T.M., Jr.; Bodhidatta, D.; Phulsuksombati, D.; Tippayachai, B.; Coleman, R.E. Short report: Variation in the 56-kD type-specific antigen gene of Orientia tsutsugamushi isolated from patients in Thailand. Am. J. Trop. Med. Hyg. 2003, 68, 299-300. [CrossRef] [PubMed]

12. Wangrangsimakul, T.; Phuklia, W.; Newton, P.N.; Richards, A.L.; Day, N.P.J. Scrub Typhus and the Misconception of Doxycycline Resistance. Clin. Infect. Dis. 2020, 70, 2444-2449. [CrossRef] [PubMed]

13. Wangrangsimakul, T.; Phuklia, W.; Newton, P.N.; Richards, A.L.; Day, N.P.J. Reply to Watt. Clin. Infect. Dis. 2020, 71, 1580-1581. [CrossRef]

14. Trent, B.; Fisher, J.; Soong, L. Scrub Typhus Pathogenesis: Innate Immune Response and Lung Injury During Orientia tsutsugamushi Infection. Front. Microbiol. 2019, 10, 2065. [CrossRef]

15. Janardhanan, J.; Joseph Martin, S.; Astrup, E.; Veeramanikandan, R.; Aukrust, P.; Abraham, O.C.; Varghese, G.M. Single-nucleotide polymorphisms in Toll-like receptor (TLR)-2, TLR4 and heat shock protein 70 genes and susceptibility to scrub typhus. J. Hum. Genet. 2013, 58, 707-710. [CrossRef] [PubMed]

16. Kim, Y.C.; Jeong, M.J.; Jeong, B.H. Strong association of regulatory single nucleotide polymorphisms (SNPs) of the IFITM3 gene with influenza H1N1 2009 pandemic virus infection. Cell Mol. Immunol. 2020, 17, 662-664. [CrossRef] [PubMed]

17. Kim, S.K.; Kim, Y.C.; Won, S.Y.; Jeong, B.H. Potential scrapie-associated polymorphisms of the prion protein gene (PRNP) in Korean native black goats. Sci. Rep. 2019, 9, 15293. [CrossRef]

18. Kim, Y.C.; Kim, S.K.; Jeong, B.H. Scrapie susceptibility-associated indel polymorphism of shadow of prion protein gene (SPRN) in Korean native black goats. Sci. Rep. 2019, 9, 15261. [CrossRef] [PubMed]

19. Won, S.Y.; Kim, Y.C.; Kim, K.; Kim, A.D.; Jeong, B.H. The First Report of Polymorphisms and Genetic Features of the prion-like Protein Gene (PRND) in a Prion Disease-Resistant Animal, Dog. Int. J. Mol. Sci. 2019, 20, 1404. [CrossRef]

20. Bush, W.S.; Moore, J.H. Chapter 11: Genome-wide association studies. Plos Comput. Biol. 2012, 8, e1002822. [CrossRef] [PubMed]

21. Tam, V.; Patel, N.; Turcotte, M.; Bosse, Y.; Pare, G.; Meyre, D. Benefits and limitations of genome-wide association studies. Nat. Rev. Genet. 2019, 20, 467-484. [CrossRef] [PubMed]

22. Kim, S.; Kim, H.; Cho, N.; Lee, S.K.; Han, B.G.; Sull, J.W.; Jee, S.H.; Shin, C. Identification of FAM13A gene associated with the ratio of FEV1 to FVC in Korean population by genome-wide association studies including gene-environment interactions. J. Hum. Genet. 2015, 60, 139-145. [CrossRef]

23. Hu, Y.; Vinayagam, A.; Nand, A.; Comjean, A.; Chung, V.; Hao, T.; Mohr, S.E.; Perrimon, N. Molecular Interaction Search Tool (MIST): An integrated resource for mining gene and protein interaction data. Nucleic Acids Res. 2018, 46, D567-D574. [CrossRef] [PubMed]

24. Tang, H.; Thomas, P.D. PANTHER-PSEP: Predicting disease-causing genetic variants using position-specific evolutionary preservation. Bioinformatics 2016, 32, 2230-2232. [CrossRef]

25. Kweon, S.S.; Choi, J.S.; Lim, H.S.; Kim, J.R.; Kim, K.Y.; Ryu, S.Y.; Yoo, H.S.; Park, O. Rapid increase of scrub typhus, South Korea, 2001-2006. Emerg. Infect. Dis. 2009, 15, 1127-1129. [CrossRef] [PubMed]

26. Jan, R.H.; Chen, C.J.; Hong, Y.R.; Lin, Y.L.; Chen, L.K. A surface antigen of Orientia tsutsugamushi activates human monocytederived dendritic cells via nuclear factor-kB and p38 mitogen-activated protein kinase pathways. Indian J. Med. Res. 2018, 148, 215-224. [CrossRef] 